\title{
Can iFOBT (immunochemical faecal occult blood test) for bowel cancer screening be safely deferred for five years after a colonoscopy?
}

\author{
Authors: Myat Myat Khaing, ${ }^{\mathrm{A}}$ Lei Lin, ${ }^{\mathrm{A}}$ Tony Rahman, ${ }^{\mathrm{A}}$ Geogry Peter-Kini, ${ }^{\mathrm{B}}$ John Croese, ${ }^{\mathrm{A}}$ Ruth Hodgson, ${ }^{\mathrm{A}}$ \\ James Thomas, ${ }^{A}$ Petrina Kellar, ${ }^{A}$ Debra Whittaker, ${ }^{B}$ Felicity Hartnell, ${ }^{B}$ Ann Vandeleur, ${ }^{A}$ Vinny Ea ${ }^{A}$ and Peter Boyd ${ }^{B}$
}

\section{Background}

Australian and international guidelines suggest that immunochemical faecal occult blood test (iFOBT) for colorectal cancer (CRC) screening may be deferred for 5-10 years after a negative colonoscopy. However, it is not uncommon for community health practitioners to repeat iFOBT at earlier than recommended intervals after a previous colonoscopy due to concerns over potential missed detection of neoplasms. The aim of this retrospective multicentre observational study was to evaluate the outcomes of patients who had a repeat colonoscopy for iFOBT positivity within five years of a previous colonoscopy showing low risk or normal findings, to assess the risk of interval CRC and advanced colorectal neoplasia (ACRN). We sought to determine if having a prior colonoscopy with normal or low risk findings within the last five years would predict for negative findings on a repeat colonoscopy in asymptomatic patients who were iFOBT positive.

\section{Methods}

We retrospectively identified patients who underwent a repeat colonoscopy for iFOBT positivity within five years of a previous colonoscopy showing normal or low risk findings from November 2016 to November 2018. Patients identified from Provation, Auscare and the Viewer databases were included from four sites, including The Prince Charles Hospital in Brisbane, Cairns Base Hospital, Innisfail Hospital and Atherton District Hospital in Northern Queensland. We excluded patients with a past history of CRC, patients with a familial malignancy syndrome, patients with poor bowel preparation and patients who were symptomatic.

\section{Result}

Among 3,795 patients who underwent colonoscopy for a positive iFOBT in the study period, 239 had a previous colonoscopy in the five-year window that met inclusion criteria. Of these, $4(1.7 \%)$ patients had locally advanced CRC at two regional sites, and 19 (7.9\%) patients had ACRN. 42 (17.6\%) patients had high risk adenoma, $21(8.8 \%)$ patients had sessile serrated adenoma and 87 (36.4\%) patients had adenoma of various sizes. Inadequate bowel preparation in the previous colonoscopy was associated with the findings of subsequent high-risk adenomas and CRC.

\section{Conclusions}

Our study observed that a substantial portion of patients have interval ACRN associated with a positive iFOBT performed outside of guidelines. These finding were not uniform between the study sites and may reflect local differences, highlighting the importance of interpretation of guidelines, the ability of the clinician to override guidelines if clinically indicated, the need for colonoscopy care standards and teamwork that may facilitate excellence in practice.

\section{Conflicts of interest}

None declared. 